\title{
Direct Evidence of Coexisting Horseshoe and Extended Helix Conformations of Membrane-Bound Alpha-Synuclein
}

\author{
Marta Robotta ${ }^{[b]}$ Patrick Braun, ${ }^{[b]}$ Bart van Rooijen, ${ }^{[c]}$ Vinod Subramaniam, ${ }^{*[c]}$ Martina Huber, ${ }^{*[a]}$ and \\ Malte Drescher ${ }^{*[b]}$ \\ $\alpha$-Synuclein $(\alpha S)$ is a 140 -residue protein abundantly present in \\ the Lewy bodies characteristic of Parkinson's disease. ${ }^{[1-3]}$ It is a \\ member of the class of intrinsically disordered proteins (IDPs) \\ that have unusual properties and whose physiological rele- \\ vance is becoming increasingly recognized. ${ }^{[4]}$ IDPs lack a well- \\ defined three-dimensional fold and display remarkable confor- \\ mational flexibility. This property potentially enables them to

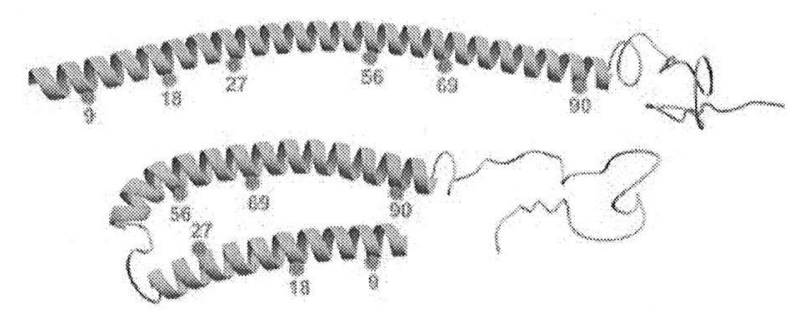
be promiscuous in their interactions and to adapt their structure according to the needed function. In the case of $\alpha \mathrm{S}$, the protein is capable of adopting a $\beta$-sheet structure in the amyloid fibrils constituting the Lewy bodies and an $\alpha$-helical structure in the membrane bound form. The exact physiological role of $\alpha \mathrm{S}$ has yet to be determined, but membrane binding seems to be important for its function. ${ }^{[5-8]}$ As a consequence, the membrane bound form has received considerable attention in the last several years.

It is generally accepted that upon binding to membranes $\alpha \mathrm{S}$ adopts an amphipathic, $\alpha$-helical structure involving residues $1-100 . .^{[9-15]}$ The exact arrangement of the helix on the membrane surface is, however, controversial. The NMR structure of $\alpha S$ bound to SDS micelles revealed a break in the helix, resulting in two antiparallel alpha-helices (horseshoe model, Figure 1, bottom). ${ }^{[13]}$ This model was confirmed by techniques based on electron paramagnetic resonance (EPR). ${ }^{[16]}$ It has been postulated that the small size of the micelles (typical di-

\section{[a] Dr. M. Huber}

Leiden Institute of Physics

University of Leiden

PO Box 9504, 2300 RA Leiden (The Netherlands)

Fax: $(+31) 715275819$

Email:mhuber@molphys.leidenuniv.nl

Homepage: www.molphys.leidenuniv.nl/monos/

[b] M. Robotta, ${ }^{+}$P. Braun, ${ }^{+}$Dr. M. Drescher

Department of Chemistry

University of Konstanz

Universitätsstr. 10, 78457 Konstanz (Germany)

Fax: $(+49) 753883139$

E mail:malte.drescher@uni konstanz.de

Homepage: www.uni konstanz.de/drescher

[c] Dr. B. van Rooijen, Prof. Dr. V. Subramaniam

Nanobiophysics, MESA +

Institute for Nanotechnology \&

MIRA Institute for Biomedical Technology and Technical Medicine

University of Twente

PO Box 217, 7500AE Enschede (The Netherlands)

Fax: $(+31) 534891105$

Email:v.subramaniam@utwente.n

Homepage: www.utwente.nl/nbp

$\left[{ }^{+}\right]$These authors contributed equally to this work.

Supporting information for this article is available on the WWW under http://dx.doi.org/10.1002/cphc.201000815.

Figure 1. NMR structure ${ }^{[13]}$ of micelle bound $\alpha S$ (bottom) and hypothetical model of an extended $\alpha$ helix (top). Spin label positions are indicated in red.

ameter $\left.5 \mathrm{~nm}^{[17]}\right)$ used in these studies may have artificially constrained the protein into a horseshoe structure, and subsequent studies were performed on small unilamellar vesicles (SUVs) and bicelles. Of the eight studies published on this issue to date, three support the horseshoe ${ }^{[18-20]}$ conformation, and five the extended $d^{[5,21-24]}$ conformation, despite using similar experimental techniques and often with only slight variations in experimental conditions. There can be several reasons for the apparent discrepancies. The protein could be sensitive to the exact experimental conditions, accessible distance ranges of the method could limit the observation to only one of the forms and incomplete binding could aggravate the uncertainty.

Herein we present evidence that the horseshoe configuration is also found on larger vesicles, and that under these conditions $\alpha \mathrm{S}$ coexists in a superposition of both horseshoe and extended forms shown in Figure 1.

The vesicles used herein were large unilamellar vesicles (LUV, $100 \mathrm{~nm}$ in diameter) of the negatively charged lipid POPG \{1-Palmitoyl-2-Oleoyl-sn-Glycero-3-[Phosphorac-(1-glycerol)]\}. It has been shown that $\alpha \mathrm{S}$ binds most effectively to negatively charged membranes consistent with the exposure of positively charged amino acid residues in the helical section of the N-terminus of $\alpha S^{[13]}$ The POPG LUVs are stable upon addition of $\alpha \mathrm{S}$ and continuous wave (cw) EPR on singly labelled $\alpha \mathrm{S}$, according to an approach described before, ${ }^{[20]}$ which showed that, under the conditions used here, quantitative binding is achieved. Quantitative binding was also confirmed by fluorescence correlation spectroscopy (FCS) [Supporting Information]. Furthermore, no evidence for aggregates or oligomers of $\alpha S$ on the membrane, such as those observed for $\alpha S$ on POPG SUVs, ${ }^{[25]}$ was found under the conditions of the present investigation (Supporting Information).

In order to monitor the structure of membrane-bound $\alpha \mathrm{S}$, a set of double cysteine mutants was specifically labelled with MTSL [1-oxyl-(2,2,5,5-tetramethylpyrroline-3-methyl)methanethiosulfonate], using the site-directed spin labelling approach 
pioneered by Hubbell et al. ${ }^{[26]}$ Spin labels were attached at positions 9 and 69 ( $\alpha S 9 / 69), \alpha S 9 / 90, \alpha S 18 / 69, \alpha S 18 / 90$, and $\alpha S 27 / 56$ as indicated in Figure 1. Distances between the spin labels were obtained by the pulsed, two-frequency EPR method known as double electron-electron resonance (DEER), ${ }^{[27]}$ which, in principle, provides access to distances between $1.5 \mathrm{~nm}$ and $8 \mathrm{~nm}^{[28]}$ Distances below $1.5 \mathrm{~nm}$ were excluded by CW EPR for all double mutants (Supporting Information). The DEER data were analyzed (Supporting Information) to extract the model-free distance distributions. For $\alpha S 9 / 69$, $\alpha S 9 / 90, \alpha S 18 / 69$, and $\alpha \mathrm{S} 18 / 90$ these distributions are well described by a single Gaussian, the parameters of which are given in Table 1. The width of the distribution results from the flexibility of the spin-label linker as well as from the conformational flexibility of the protein. The distances obtained for these four mutants are in good agreement with the horseshoe model. The extended conformation should give distances of $8 \mathrm{~nm}$ or larger.

In contrast to the other mutants, the DEER time trace (Figure $2 \mathrm{~A}$ ) for the $\alpha \mathrm{S} 27 / 56$ mutant cannot be described by a single Gaussian. A model-free analysis using Tikhonov regularisation results in the distance distribution depicted in Figure 2B (black line), which clearly consists of two contributions (see the Supporting Information for the L-curve). This model-free distance distribution is well described by two Gaussians, and fits of the DEER time traces with a superposition of two Gaussian distance distributions lead to similar final parameters (Supporting Information), confirming that the analysis is model-independent. The shorter-distance Gaussian agrees well with the expected distance of $2.7 \mathrm{~nm}$ for the horseshoe conformation derived from the NMR structure (pdb access code $1 \mathrm{XQ8})^{[13]}$ while the longer-distance Gaussian is consistent with the $4.9 \mathrm{~nm}$ expected for an extended $\alpha$-helix. This can only be explained if horseshoe and extended forms coexist under the membrane conditions employed herein.

To determine how this observation ties in with the results on the remaining mutants, we analyzed the fraction of spin pairs, which is obtained from the modulation depth of the DEER time trace. The fraction of spins obtained from mutants $\alpha S 9 / 69, \alpha S 9 / 90, \alpha S 18 / 69$, and $\alpha S 18 / 90$ (Supporting Information) is significantly smaller than $100 \%$, whereas for $\alpha \mathrm{S} 27 / 56$

\begin{tabular}{|c|c|c|c|c|c|}
\hline $\begin{array}{l}\alpha S \text { double } \\
\text { Cys mutant }\end{array}$ & $\begin{array}{l}\text { Distance } \\
{[\mathrm{nm}]}\end{array}$ & $\begin{array}{l}\text { FWHM of } \\
\text { distribution } \\
{[\mathrm{nm}]}\end{array}$ & $\begin{array}{l}\text { Fraction of } \\
\alpha S \\
\text { contributing } \\
\text { to distance }\end{array}$ & $\begin{array}{l}\text { Horseshoe model: } C_{\beta} C_{\beta} \\
\text { distance }[\mathrm{nm}] \\
1 \mathrm{XQ} 8^{[13]}\end{array}$ & $\begin{array}{l}\text { Extended helix model: } \\
\mathrm{C}_{\beta} \mathrm{C}_{\beta} \text { distance }{ }^{[\mathrm{b}]} \\
{[\mathrm{nm}]}\end{array}$ \\
\hline $9 / 69$ & $3.7 \pm 0.2$ & $1.8 \pm 0.2$ & & 2.7 & 9 \\
\hline 9/90 & $3.5 \pm 0.1$ & $1.6 \pm 0.2$ & & 2.1 & 12 \\
\hline $18 / 69$ & $2.7 \pm 0.1$ & $0.95 \pm 0.05$ & & 2.8 & 8 \\
\hline $18 / 90$ & $3.3 \pm 0.1$ & $1.4 \pm 0.2$ & & 3.7 & 11 \\
\hline \multirow[t]{2}{*}{$27 / 56^{[a]}$} & $2.7 \pm 0.1$ & $0.55 \pm 0.05$ & $20 \% \pm 5 \%$ & 2.7 & \\
\hline & $4.3 \pm 0.1$ & $0.95 \pm 0.05$ & $80 \% \pm 5 \%$ & & 4.9 \\
\hline
\end{tabular}
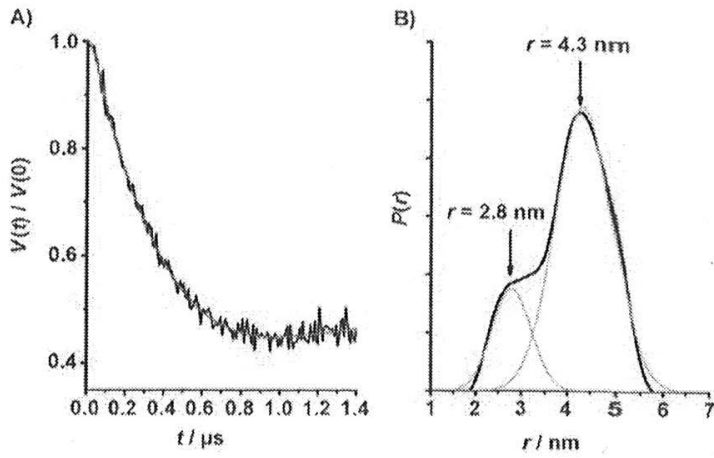

Figure 2. A) DEER time trace after background correction for $\alpha \mathrm{S} 27 / 56$ bound to POPG LUV (black) and fit (blue) corresponding to the distance dis tribution shown in: B) Distance distribution derived model free by Tikhonov regularisation (black) described by two Gaussians (green).

the two Gaussian distance distributions combine to almost $100 \%$. Thus it is only for this mutant, for which two distances are observed and attributed to the horseshoe and extended forms that the modulation depth accounts for all protein present. We therefore conclude that for the first four mutants a significant fraction of the proteins are in the extended form, ${ }^{[5 ; 22-24]}$ and therefore escape detection. The amount of these "missing spins" is notoriously difficult to quantify. In addition to the proteins in the extended form, that is, with a distance larger than the measurement range, this population also contains proteins that have only one functional spin label and possibly $\alpha \mathrm{S}$ that is not bound to the membrane (Supporting Information). Even under our conditions, an uncertainty of $15 \%$ in these values cannot be avoided (Supporting Information), underlining the need to measure both conformations, which is only possible for the $\alpha \mathrm{S} 27 / 56$ mutant in our case.

The presence of the horseshoe conformation on the LUVS revealed by all five mutants investigated herein clearly shows that this conformation is stable on intact large vesicles, and is therefore not likely to be an artifact induced by the small size of SUVS or micelles. Consequently it can be considered a physiologically relevant structure on two-dimensional membranes. The fraction of the horseshoe conformation is significantly smaller than that of the extended conformation, however, which reveals a preference for the extended form. We have previously speculated that the horseshoe conformation could be linked to aggregation of $\alpha \mathrm{S}$ on the membrane. ${ }^{[25]}$ Herein we did not find signatures of aggregation, but the small fraction of proteins in the horseshoe conformation would be below the detection limit for aggregates, so we cannot exclude that aggregation accompanies the horseshoe form found on the LUVs used here. 
The coexistence of horseshoe and extended forms on POPGSUVs found herein casts a new light on the debate about the horseshoe and extended forms of membrane-bound $\alpha \mathrm{S}$. Since both forms coexist under the conditions employed here, it is reasonable to infer that only small changes are needed to tip the balance one way or the other, suggesting that the conformation could be a subtle function of the experimental conditions. Therefore, different lipids, vesicle sizes, or even subtle differences in preparation protocols may easily shift the equilibrium, providing a rationale for the different results found in previous studies. ${ }^{[5,18-24]}$ The $\alpha \mathrm{S} 27 / 56$ variant is ideally suited to investigate this question in the future. Another factor in the discrepancy is that the analysis of the data can be affected by the sensitivity of the method to different distance regimes, especially in cases where the distance of one conformation is outside the measurement range. Further uncertainties can arise from incomplete binding of the protein to the membrane. Finally, while previous studies show the interconversion of the horseshoe and extended form (see ref. [29] and references therein), the simultaneous presence of both forms emphasizes the flexibility of $\alpha \mathrm{S}^{1}$

The results obtained herein demonstrate that the challenges of structure determination for intrinsically disordered proteins derive not only from the flexibility of these proteins, but also from the sensitivity of the structures to subtle details of the environment. We speculate that for $\alpha \mathrm{S}$ on membranes, the range of conformations found is not an artifact, but a manifestation of the energetic proximity of these conformations, an attribute that could be relevant for the function of $\alpha S$ on the membrane.;

\section{Experimental Section}

DEER experiments were performed using a Bruker Elexsys E580 XBand spectrometer. Data were analysed using DEERAnalysis 2008.1 and model-free Tikhonov regularization. ${ }^{[1,32]}$ For details of sample preparation, measurements and analysis, see the Supporting Information.

\section{Acknowledgements}

This work was financially supported by Deutsche Forschungsgemeinschaft (DR 743/2-1), by the Dutch science foundations N.W.O. and FOM, and by the Nanotechnology network in the Netherlands, NanoNed, project 7921. We thank Christian Jüngst for experimental contributions.

\footnotetext{
While this manuscript was under review, a study revealing that the coexis tence of both forms also pertains to $\alpha S$ on SDS micelles has appeared. It does so with DEER data reaching out to very long distances. ${ }^{[30]}$
}

Keywords: electron paramagnetic resonance - membranes proteins $\cdot$ site-directed spin labeling $\cdot$ vesicles

[1] M. G. Spillantini, M. L. Schmidt, V. M. Y. Lee, J. Q. Trojanowski, R. Jakes, M. Goedert, Nature 1997, 388, 839840.

[2] M. Goedert, Nat. Rev. Neurosci. 2001, 2, 492501.

[3] P. H. Weinreb, W. G. Zhen, A. W. Poon, K. A. Conway, P. T. Lansbury, Bio chemistry 1996, 35, 1370913715.

[4] P. Tompa, Structure and Function of Intrinsically Disordered Proteins, CRC Press, Boca Raton, 2009.

[5] R. Bussell, Jr., T. F. Ramlall, D. Eliezer, Protein Sci. 2005, 14, 862872

[6] K. K. Dev, K. Hofele, S. Barbieri, V. L. Buchman, H. van der Putten, Neuro pharmacology 2003, 45, 1444.

[7] J. Lotharius, P. Brundin, Hum. Mol. Genet. 2002, 11, 23952407.

[8] D. D. Murphy, S. M. Rueter, J. Q. Trojanowski, V. M. Lee, J. Neurosci. 2000, 20, 32143220

[9] W. S. Davidson, A. Jonas, D. F. Clayton, J. M. George, J. Biol. Chem. 1998, 273, 94439449.

[10] R. J. Perrin, W. S. Woods, D. F. Clayton, J. M. George, J. Biol. Chem. 2000, 275, 3439334398.

[11] A. C. M. Ferreon, A. A. Deniz, Biochemistry 2007, 46, 44994509.

[12] M. Ramakrishnan, P. H. Jensen, D. Marsh, Biochemistry 2003, 42, 12919 12926.

[13] T. S. Ulmer, A. Bax, N. B. Cole, R. L. Nussbaum, J. Biol. Chem. 2005, 280, 95959603.

[14] C. C. Jao, A. Der Sarkissian, J. Chen, R. Langen, Proc. Natl. Acad. Sci. USA 2004, 101, 83318336.

[15] J. C. Lee, R. Langen, P. A. Hummel, H. B. Gray, J. R. Winkler, Proc. Natl. Acad. Sci. USA 2004, 101, 1646616471.

[16] P. Borbat, T. F. Ramlall, J. H. Freed, D. Eliezer, J. Am. Chem. Soc. 2006 , 128, 1000410005.

[17] R. Itri, L. Q. Amaral, J. Chem. Phys. 1991, 95, 423427.

[18] M. Bortolus, F. Tombolato, I. Tessari, M. Bisaglia, S. Mammi, L. Bubacco, A. Ferrarini, A. L. Maniero, J. Am. Chem. Soc. 2008, 130, 66906691.

[19] S. Chandra, X. C. Chen, J. Rizo, R. Jahn, T. C. Sudhof, J. Biol. Chem. 2003, 278, 1531315318.

[20] M. Drescher, G. Veldhuis, B. D. van Rooijen, S. Milikisyants, V. Subrama niam, M. Huber, J. Am. Chem. Soc. 2008, 130, 77967797.

[21] A. Trexler, E. Rhoades, Biochemistry 2009, 48, 23042306.

[22] R. Bussell, Jr., D. Eliezer, J. Mol. Biol. 2003, 329, 763778.

[23] E. R. Georgieva, T. F. Ramlall, P. P. Borbat, J. H. Freed, D. Eliezer, J. Am. Chem. Soc. 2008, 130, 1285612857.

[24] C. C. Jao, B. G. Hegde, J. Chen, I. S. Haworth, R. Langen, Proc. Natl. Acad. Sci. USA 2008, 105, 1966619671.

[25] M. Drescher, B. D. van Rooijen, G. Veldhuis, V. Subramaniam, M. Huber, J. Am. Chem. Soc. 2010, 132, 40804081.

[26] W. L. Hubbell, C. Altenbach, Curr. Opin. Struct. Biol. 1994, 4, 566573.

[27] M. Pannier, S. Veit, A. Godt, G. Jeschke, H. W. Spiess, J. Magn. Reson. 2000, 142, 331340.

[28] A. Godt, M. Schulte, H. Zimmermann, G. Jeschke, Angew. Chem. 2006 118, 7722 7726; Angew. Chem. Int. Ed. 2006, 45, 75607564

[29] A. C. M. Ferreon, C. R. Moran, J. C. Ferreon, A. A. Deniz, Angew. Chem 2010, 122, 3547 3550; Angew. Chem. Int. Ed. 2010, 49, 34693472.

[30] E. R. Georgieva, T. F. Ramlall, P. P. Borbat, J. H. Freed, D. Eliezer, J. Biol. Chem. 2010, 285, 2826128274.

[31] G. Jeschke, A. Koch, U. Jonas, A. Godt, J. Magn. Reson. 2002, 155, 72 82.

[32] G. Jeschke, Macromol. Rapid Commun. 2002, 23, 227246. 